\title{
INVENTAIRE QUALITATIF DE L'INSTRUMENTATION UTILISÉE EN ENVIRONNEMENT LITTORAL
}

\author{
M. PAILLARD (*) et G. CLAVERIE (**)
}

(*) IFREMER, DITI/GO/SOM, B.P. 70, 29280 Plouzané

(**) CREOCEAN, Allée des Tamaris, 17000 La Rochelle

\begin{abstract}
The "Direction de l'Ingenierie, de la Technologie et de I'Informatique" (DITI) of IFREMER has undertaken an investigation concerning the instruments used for in-situ measurements and samplings in coastal environment studies. The investigation was carried out by a subsidiary of IFREMER, CREOCEAN. Its aim was to establish a qualitative catalog of the instruments and especially to inventory their deficiencies resulting either from misconception or misuse. A list of equipments to be submitted to a thorough examination or to a program of development, modification and calibration has been established.

The investigations carried out among the users and the suppliers have been done simultaneously. The increasing number of studies concerning coastal environment and the development of means of measurement has pushed us to associate every experience, and to join all the means and competences in order to put on the market reliable and well-adapted equipment.
\end{abstract}

\section{PRESENTATION}

La Direction de l'Ingénierie, de la Technologie et de I'Informatique (DITI) de I'TFREMER a effectué une enquête auprès des utilisateurs et des fournisseurs de matériels de prélèvement et de mesure in-situ utilisés en Environnement Littoral. Cette enquête, réalisée avec sa filiale CREOCEAN, était destinée à établir un catalogue qualitatif des matériels. Il s'agissait, notamment, d'en recenser les éventuels défauts, de conception ou de mise en oeuvre préjudiciables à la qualité de la donnée, identifiés par les utilisateurs afin d'y remédier.

\section{OBJECTIFS}

L'étude a été volontairement limitée aux appareils utilisables en environnement littoral sur des fonds compris entre 0 et $100 \mathrm{~m}$, estran compris dans le cas où les appareils ont une spécificité marine.

Les différentes phases de l'étude doivent permettre d'atteindre les objectifs suivants :

* recenser les matériels standards disponibles sur le marché,

* établir un inventaire qualitatif de l'instrumentation utilisé :

- pour les matériels standards des fournisseurs,

- pour les matériels développés spécifiquement par les utilisateurs,

* définir la qualité des prélèvements et des mesures en milieu naturel (in situ) en fonction des différents appareils (impact des appareils et de leur mise en oeuvre sur la qualité de la donnée),
* identifier des défauts de conception ou de mise en oeuvre préjudiciables à la qualité des données,

* identifier les demandes consensuelles concernant les améliorations ou développements,

* identifier les développements en cours,

* identifier les besoins des utilisateurs en calibration, validation et collaboration,

* sélectionner des appareils à soumettre à une analyse critique plus détaillée.

\section{METHODOLOGIE}

Cette étude a nécessité un important travail de recherche afin de lister de façon la plus exhaustive possible les utilisateurs et les fournisseurs. Les enquêtes auprès des fournisseurs et des utilisateurs ont été effectuées simultanément à partir d'un vaste mailing suivi de relances et de contacts téléphoniques.

Le recensement des matériels disponibles sur le marché a été réalisé pour vérifier l'adéquation entre l'offre des foumisseurs et les demandes des utilisateurs.

\section{Enquète "Fournisseurs"}

Un mailing a été adressé à plus de 200 fournisseurs d'instrumentation pour l'environnement littoral et le Génie Côtier (5 en France, 49 en Grande Bretagne, 41 aux USA, 48 dans les autres pays d'Europe et 11 dans les autres pays). Plus de la moitié a répondu. Les documents transmis par les sociétés ont permis de recenser environ 500 produits différents.

Une classification des différents matériels a été mise en place pour faciliter l'utilisation de cet inventaire (Résultats classés et archivés sur une base de données).

\section{Enquète "Utilisateurs"}

Un premier courrier a été adressé aux laboratoires et organismes recensés. Il comportait un questionnaire de 6 pages, accompagné d'une lettre définissant les objectifs à atteindre et les moyens mis en oeuvre pour cette étude.

Ce courrier adressé aux utilisateurs devait leur permettre de :

* porter un regard critique sur les matériels qu'ils utilisent (à l'aide du questionnaire basé sur des indices de satisfaction),

* décrire les matériels développés spécialement par leur laboratoire.

Plus de 300 laboratoires et organismes ont été ainsi contactés pour un retour d'environ 30\%. Les réponses ont permis de rédiger un "catalogue" de matériels, mais les résultats obtenus, par rapport à l'objectif principal (impact de la conception ou de la mise en oeuvre sur la 
qualité de la donnée) ne faisaient pas apparaître de critiques consensuelles.

Toutefois, ce premier inventaire a fourni une vue d'ensemble dans des domáines très divers. L'identification des đéveloppements réalisés a été fructueuse et a apporté des renseignements intéressants sur les besoins des utilisateurs et sur les lacunes des produits standards.

Cette première phase de l'enquête a montré également les limites d'un questionnaire rigide. Il est apparu nécessaire de poursuivre ce travail à partir d'un complément d'enquête s'orientant vers une recherche plus systématique des demandes et des besoins de utilisateurs dans le but de mettre en évidence les insatisfactions sur les matériels existants ; mauvaise qualité des prélèvements et des mesures, problèmes de fiabilité, de reproductibilité, perturbation du milieu, etc.

In fine, près de 400 utilisateurs (potentiels) ont été contactés pour ces enquêtes. 35\% d'entre eux ont répondu. Ainsi, près de 100 matériels standards couramment utilisés et 75 développements spécifiques ont été recensés.

\section{RESULTATS}

L'analyse faite à l'issue de ce travail d'enquête auprès des utilisateurs de matériels de mesure et de prélèvement pour l'environnement littoral est qu'il n'apparâit pas de critiques "consensuelles" sur les matériels standards en ce qui concerne la "qualité de la donnée". Cela malgré un nombre important de remarques.

En revanche, les nombreux besoins exprimés par les utilisateurs peuvent être identifiés comme un manque ou une insatisfaction dans les matériels standards.

L'importance du nombre de matériels développés par les laboratoires, en regard de celui des matériels standards réellement utilisés, montre des lacunes dans l'offre des fournisseurs. Cet état de fait est essentiellement lié à l'étroitesse de ce marché.

Enfin, le nombre d'utilisateurs ayant exprimé un besoin similaire représente une bonne indication sur les domaines d'intérêt prépondérants. Cela permet de dégager des axes de travail pour la suite de cette étude.

Les besoins les plus couramment exprimés peuvent se résumer comme suit :

* besoins en centrales autonomes pour l'acquisition de données.

Sous l'appellation "Centrale d'acquisition" sont regroupés des appareils très différents, correspondant à des besoins très spécifiques. Chaque application implique des contraintes différentes; autonomie, capacité de stockage, nombre de capteurs, adaptabilité, volume, poids,...

* besoins en appareillages benthiques autonomes de toutes sortes allant du très simple au plus complexe. Certaines demandes portent sur l'automatisation de chambres benthiques existantes. Ces types de matériels cadrent bien avec les besoins actuels en environnement littoral et l'objectif de cette étude (analyse de l'impact de l'appareil sur la mesure). Ils sont utilisés pour :

- l'analyse des flux à l'interface eau / sédiment sédiment

- les mesures hydrodynamiques à l'interface eau /

- les mesures rhéologiques à l'interface eau / sédiment
* besoins en engins autonomes de prélèvement.

Il s'agit de matériels permettant de limiter le caractère aléatoire et ponctuel des prélèvements opérés manuellement. Le but étant de multiplier le nombre de prélèvements sans contrainte de temps ni de personnel.

* besoins en carottiers de petite taille

Ces besoins correspondent à la fois aux carottiers multitubes et aux carottiers adaptés aux contraintes du côtier.

* besoins en validation et intercalibration

Ces types de demandes cadrent bien avec lobjectif initial de cette étude. La réalisation de certaines mesures est souvent délicate et il est clair que l'appareillage utilisé (ainsi que sa mise en oeuvre) peut avoir un impact considérable sur la précision des mesures. Les mesures les plus citées sont :

- sondes CTD (pression, température, conductivité),

- mesures de flux aux interfaces

- mesure de houle en zone côtière. L'agitation littorale apparaît plus difficile à mesurer que la houle au large (hauteur et période plus faible), mais son évaluation est pourtant nécessaire dans de nombreux cas.

* quelques autres thèmes (bouteilles de prélèvements, mesures géotechniques, pièges à particules dérivants, etc.)

\section{CONCLUSION}

La deuxième phase d'enquête a atteint les objectifs fixés. Les différents besoins exprimés ont permis de mettre en avant quelques types de matériels (ainsi que quelques méthodes de mesure) qui peuvent être soumis à une analyse plus poussée.

Ces types de besoins en matériels ou études étaient, pour la plupart, identifiés à l'IFREMER, mais principalement à partir de demandes ponctuelles. Quelques matériels ont été développés (chambres benthiques). Des intercalibrations sont effectués également régulièrement (sondes CTD).

Mais, cette étude a permis de regrouper et classer des besoins exprimés par la communauté scientifique nationale. Elle a permis également d'identifier des demandes de collaboration.

Il apparaît que l'on peut classer les besoins des utilisateurs suivant 2 groupes prioritaires principaux :

- mesures et prélèvements aux interfaces eau / sédiment

- intercalibration /validation de matériels existants

La phase actuelle de ce programme porte essentiellement sur ces deux priorités. C'est, d'une part, l'élaboration de cahiers des charges de matériels de mesure et de prélèvement dans les sédiments fins cohésifs et d'autre part, l'élaboration et la mise en oeuvre de protocoles d'intercalibrations de certains matériels (mesures de houle par faibles fonds). 